\title{
Assessment battery for communication (ABaCo): normative data
}

\author{
Romina Angeleri - Francesca M. Bosco • \\ Ilaria Gabbatore • Bruno G. Bara • Katiuscia Sacco
}

Published online: 17 December 2011

(C) Psychonomic Society, Inc. 2011

\begin{abstract}
The Assessment Battery for Communication $(\mathrm{ABaCo})$ was introduced to evaluate pragmatic abilities in patients with cerebral lesions. The battery is organized into five evaluation scales focusing on separate components of pragmatic competence. In the present study, we present normative data for individuals $15-75$ years of age $(N=$ 300). The sample was stratified by age, sex, and years of education, according to Italian National Institute of Statistics indications in order to be representative of the general national population. Since performance on the $\mathrm{ABaCo}$ decreases with age and lower years of education, the norms were stratified for both age and education. The $\mathrm{ABaCo}$ is a valuable tool in clinical practice; the normative data provided here will enable clinicians to determine different kinds and specific levels of communicative impairments more precisely.
\end{abstract}

Keywords Normative data - Communication - Assessment . Pragmatics

R. Angeleri $(\bowtie) \cdot$ I. Gabbatore

Center for Cognitive Science, Department of Psychology,

University of Turin,

Via Po 14

10123 Turin, Italy

e-mail: romina.angeleri@unito.it

F. M. Bosco • B. G. Bara $\cdot$ K. Sacco

Center for Cognitive Science, Department of Psychology,

University of Turin, and Neuroscience Institute of Turin,

Turin, Italy

K. Sacco

CCS fMRI Neuroradiology at Koelliker Hospital,

Turin, Italy

\section{Introduction}

The Assessment Battery for Communication (ABaCo; Sacco et al., 2008) is a clinical tool for evaluating pragmatic abilities in patients with brain injuries or other neuropsychological disturbances. The purpose of this article is to provide normative data in order to clarify and quantify communicative impairments resulting from various kinds of cerebral lesions. Normative data for a test battery provide the range of scores obtained by a group of neurologically healthy individuals with specific demographic characteristics against which to compare patients' scores (for a comprehensive discussion, see Mitrushina, Boone, Razani, \& D'Elia, 2005). The comparison between patients' performance and scores obtained by a normative sample represents a starting point in the evaluation of communicative impairments; clinicians who utilize normative data will benefit from a deeper understanding of patients' baseline abilities at the beginning of their clinical path to recovery.

The assessment of pragmatic abilities emerged as a central issue in the evaluation of patients with communicative impairments and related disorders in the early 1980s (e.g., Prutting, 1982), and the influence of pragmatic variables in treatment plans and goals has been more fully appreciated in the last 30 years. Pragmatic ability refers to a wide range of communicative behaviors concerning the way language is used in context to convey meanings (Adams, 2002; Bates, 1976; Kempson, 1975), and in the population of individuals with cerebral lesions, numerous patients have been found to have difficulties that lie principally with pragmatics. Patients typically show poor turn-taking skills and difficulty with topic maintenance, have problems understanding discourse and nonliteral meanings, and may find it difficult to interpret subtle meanings or idiomatic statements and make knowledge-based inferences in social scripts (e.g., Dennis \& 
Barnes, 1990; Friedland \& Miller, 1998; McDonald, 1993). Moreover, people with brain injuries often demonstrate normal basic linguistic skills but have difficulty adapting their communication to specific contexts (e.g., different social situations or different communicative partners) and managing complex pragmatic phenomena, such as irony and deceit (Angeleri et al., 2008; Bara, Tirassa, \& Zettin, 1997; Cutica, Bucciarelli, \& Bara, 2006). The recognition of pragmatic components as a crucial feature in rehabilitation programs thus led to the need to deal with the pervasiveness of these communicative disorders and the consequent social isolation suffered by brain-injured individuals. Importantly, in a 2-year follow-up study, Snow, Douglas, and Ponsford (1998) showed that pragmatic disorders do not spontaneously improve over time, providing evidence that communicative difficulties do not resolve as a consequence of recovery over time or speech-language input. These findings suggest that careful efforts should be made to identify and manage pragmatic disorders early on following brain injury.

Pragmatic assessment tools are still scarce in the clinical research literature, despite widespread acknowledgement of their importance (e.g., Turkstra, McDonald, \& Kaufmann, 1995). Most of the pragmatic tools that have been used for patients with brain injury and related communicative disorders focus on the assessment of specific behaviors and include inventories, rating scales, and questionnaires completed by clinicians. Examples are the Pragmatic Protocol (Prutting \& Kirchner, 1987), the Profile of Communicative Appropriateness (Penn, 1985), and, for children, the Children's Communication Checklist (Bishop, 1998). These clinical tools have the considerable advantage of involving observation of naturally occurring conversations but are also susceptible to substantial subjectivity in terms of their scoring procedures and require clinicians to undergo long periods of training. In other cases, pragmatic tests apply only to specific pathologies, such as the Right Hemisphere Communication Battery (Gardner \& Brownell, 1986), the Right Hemisphere Language Battery (Bryan, 1995) and the Awareness of Social Inference Test (McDonald, Flanagan, Rollins, \& Kinch, 2003), the first two designed for patients with right-hemisphere damage and the latter for those with traumatic brain injury.

Besides, only a few studies have been conducted in order to provide normative data for the above-mentioned tools. To the best of our knowledge, only the Right Hemisphere Communication Battery has been standardized for the Italian population in Zanini and Bryan (2003) and in Zanini, Bryan, De Luca, and Bava (2005), while no normative data are available for the other assessment batteries.

In the present article, we wish to address an important clinical issue by providing normative data against which to compare patients' performance. Cerebral lesions may affect different aspects of communicative ability, such as prosody, discourse, gesture, and nonliteral language. Individuals with cognitive-communication deficits secondary to a traumatic brain injury (TBI) or to a cerebral vascular accident may present one or more communication impairments and, thus, be grouped according to their specific profile (e.g., Ferré, Ska, Lajoie, Bleau, \& Joanette, 2011). Moreover, TBI is a heterogeneous disorder, and subsets of TBI patients have been observed to display a range of communication impairments that fall into several distinct categories (e.g., Hartley \& Jenson, 1992). Having access to normative data may facilitate clinical interpretation of individual performance on different communicative components; this seems crucial, especially because there have been reports of dissociations among different pragmatic behaviors (i.e., nonverbal communication, conversational ability, message repair, and cohesiveness of narratives; see Ehrlich \& Sipes, 1985).

Finally, normative data offer the opportunity to operationalize pragmatic functioning within a normal range and obtain a precise evaluation of domains of impairment, a crucial step in planning clinical pathways of recovery.

\section{ABaCo: the assessment battery for communication}

The Assessment Battery for Communication $(\mathrm{ABaCo})$ is an individually administered test battery designed to evaluate a patient's pragmatic ability following brain damage or other acquired/congenital neuropsychological disorders (e.g., head injury, autism, or schizophrenia). The $\mathrm{ABaCo}$ was created and developed within the framework of the cognitive pragmatics theory (Bara, 2010). It measures a wide range of communicative skills on five evaluation scales: linguistic, extralinguistic, paralinguistic, context, and conversational.

The $\mathrm{ABaCo}$ can be used to identify specific levels of impairment and provides a comprehensive assessment of the patient's pragmatic abilities and deficits in order to guide individualized rehabilitation programs.

The $\mathrm{ABaCo}$ consists of the following five evaluation scales:

1. Linguistic scale. The linguistic scale assesses the comprehension and production of communication acts expressed primarily through linguistic means. It comprises the following tasks: basic communication acts (assertions, questions, requests, and commands), standard communication acts, and nonstandard communication acts (deceits and ironies). This scale includes both in vivo participant-examiner interactions and short videos with comprehension/production tasks. In the comprehension tasks, patients have to understand the communicative interaction shown in the videos, while in the production tasks, they have to complete the communicative interaction with an appropriate communication act. 
2. Extralinguistic scale. The extralinguistic scale assesses the comprehension and production of communication acts expressed through the gesture modality only. This scale includes the same communication acts as those investigated in the linguistic scale (basic communication acts, standard and nonstandard communication acts). Also in this case, there are both in vivo interactions with the examiner and videotaped stimuli scenes for assessing comprehension and production abilities.

3. Paralinguistic scale. The paralinguistic scale assesses the comprehension and production of those elements that typically accompany speech, such as gesticulation, facial expression, and prosody. This scale includes the following tasks: basic communication acts (assertions, questions, requests, and commands), communication acts expressing an emotion (anger, happiness, fear, and sadness), and communication acts characterized by a paralinguistic contradiction (i.e., acts in which the linguistic content is in contrast with the accompanying paralinguistic indicators). The comprehension of basic communication acts and basic emotions is assessed through videotaped scenes; patients have to understand the type of communication act produced by the actors using paralinguistic indicators, choosing from among four possible alternatives provided by the examiner. The comprehension of paralinguistic contradiction is assessed through short videos in which an actor verbally expresses something in contrast with the paralinguistic indicators (e.g., facial expression); the patients have to understand the actor's actual mental states by going beyond the mere linguistic content. The production of paralinguistic aspects is assessed by asking participants to produce basic communication acts and basic emotions, using the appropriate paralinguistic indicators.

4. Context scale. The context scale assesses the adequacy/ inadequacy of communication acts with respect to discourse norms (Grice's maxims, assessed only in comprehension) and social norms (i.e., the ability to recognize whether and why communication acts are adequate as regards the given context or situation). The comprehension of discourse and social norms is assessed through videotaped scenes, while the production of social norms is assessed through in vivo interactions with the examiner, in which patients are asked to produce communication acts requiring different degrees of formality/informality. More precisely, the examiner provides some specific semantic contents and asks the participants to modulate these according to different social contexts.

5. Conversational scale. The conversational scale assesses the ability to hold a conversation, follow thread topics, and comply with turn-taking rules. To assess their conversational skills, the examiner involves patients in four different short conversations focused on a simple topic (i.e., free-time activities, favorite TV shows), each lasting about 5-6 $\mathrm{min}$.

Appendix 1 shows the number and type of items for each scale. A complete description of the assessment battery can be found in Sacco et al. (2008); here, we have included some examples of item types in Appendix 2.

The whole assessment battery can be administered in about $90 \mathrm{~min}$. However, it may be divided and administered during customized clinical sessions; each evaluation scale is independent and aims to investigate specific communicative modalities, and each scale is, in turn, divided into comprehension and production tasks. In this way, clinicians can choose from among different administration procedures, adapting these appropriately according to their patients' specific features (e.g., language functioning). The $\mathrm{ABaCo}$ is available on a CD-ROM, which means that it is very easy to use, flexible, and adaptable from time to time.

The $\mathrm{ABaCo}$ has been used for research purposes in a number of sample groups with certain medical conditions, such as traumatic brain injury patients (Angeleri et al., 2008), proving to be a useful tool for identifying communicative deficits in individuals with neuropsychological disorders. Furthermore, the $\mathrm{ABaCo}$ has been shown to have good construct validity, high interrater agreement, and good internal consistency (Sacco et al., 2008).

From a clinical viewpoint, the $\mathrm{ABaCo}$ can be profitably administered to patients with communicative disorders by a wide range of clinicians (e.g., clinical neuropsychologists, psychologists, speech-language pathologists), and coding patients' responses requires little training. There is a special user manual on how to administer the battery and assign scores. The manual provides information about test rationale and use, the theoretical framework, and evidence of reliability and validity, as well as detailed instructions for administering and scoring tasks. The CD-ROM also contains an interactive tutorial software to facilitate the learning process, provides specific examples of scoring, and illustrates possible problematic aspects of administration. The normative data presented in the present study will be included in the next version of the user manual, in order to provide clinicians with a complete evaluation tool.

\section{Method}

\section{Participants}

The normative sample consisted of volunteers recruited on the basis of notices published in a number of local high schools and university faculties, social organizations, sports clubs, and community centers for the elderly in various regions of Italy. The sample was stratified by age and years of education according to Italian National Institute of 
Statistics (ISTAT) indications in order to be representative of the general national population. None of the participants had a history of significant neurological and/or psychiatric disorders or drug or alcohol abuse. The participants were 300 normal adults (150 females and 150 males), ranging from 16 to 73 years of age $(M=44.4, S D=16.4)$. Their education ranged from 5 to 17 years of schooling $(M=10.4, S D=$ 3.97). Table 1 summarizes the distribution of individuals within each age and education group. The interaction of group (15-34 years of age) and 5 years of education is not represented, since no potential individuals met the criteria in conformity with Italian Law No. 1859/62-Italian Ministerial Decree 24.4.1963, regulating compulsory school attendance. The participants were informed about the research procedure, gave their informed consent, and volunteered to take part in the study.

\section{Materials}

\section{ABaCo (Assessment Battery for Communication)}

The $\mathrm{ABaCo}$ (Sacco et al., 2008) is a 180 -item battery focused on pragmatic assessment: 72 items were based on the examiner's prompt, using a tester-participant dialogue format in order to create interactive situations eliciting specific communicative behaviors in a natural way, and 108 were based on videotaped scenes, each lasting 20-25 s. The battery assessed both comprehension and production abilities; in particular, the videotaped scenes assessing the comprehension side showed two actors involved in a communicative interaction that participants had to understand, while the scenes assessing production showed a communicative interaction that participants had to complete with an appropriate communication act. The battery included the five above-mentioned evaluation scales: (1) linguistic, (2) extralinguistic, (3) paralinguistic, (4) context, and (5) conversational. Each scale was divided into two subscales (comprehension and production), except for the conversational scale, where comprehension and production were concurrent. Overall, the $\mathrm{ABaCo}$ thus included nine subscales: (1) linguistic comprehension, (2) linguistic production, (3) extralinguistic comprehension,

Table 1 Distribution of participants by age and education group

\begin{tabular}{lllll}
\hline $\begin{array}{l}\text { Education } \\
\text { (years of schooling) }\end{array}$ & \multicolumn{3}{l}{ Age Group } & \\
\cline { 2 - 5 } & $15-34$ & $35-54$ & $55-75$ & Total \\
\hline 5 & - & 6 & 45 & 51 \\
8 & 39 & 50 & 20 & 109 \\
13 & 45 & 35 & 15 & 95 \\
17 & 15 & 15 & 15 & 45 \\
Total & 99 & 106 & 95 & 300 \\
\hline
\end{tabular}

(4) extralinguistic production, (5) paralinguistic comprehension, (6) paralinguistic production, (7) context comprehension, (8) context production, and (9) conversational.

\section{Scoring procedure}

Two independent judges, blind with respect to the aims of the research, coded the participants' answers offline. The two judges kept the scores on specific score sheets while watching the participants' video-recorded experimental sessions. Possible scores for each task were 0 or 1: A score of 1 was awarded for correct answers, and a score of 0 for incorrect answers (for a detailed description of scoring criteria, see Angeleri et al., 2008; Sacco et al., 2008).

Procedure

The participants completed the $\mathrm{ABaCo}$ tasks individually with a research assistant at home; the experimental session usually took about $1 \mathrm{~h}$. The participants were video-recorded during the administration of the battery to enable offline scoring. Two independent judges blind to the aims of the present study rated the participants' responses. The participants were told that their participation was voluntary and that the aim and content of the research would be explained at the end of the experimental session. Informed consent was obtained from each participant. The Local Research Ethics Committee approved this study.

\section{Results}

Table 2 reports the means, standard deviations, median, minimum-maximum values, skewness, and kurtosis for age, education, and scores on the $\mathrm{ABaCo}$ subscales for each of the normative groups.

As is apparent in Fig. 1, which summarizes the distribution of scores across the nine $\mathrm{ABaCo}$ scales, scores in the normative sample were concentrated in the upper section of the range because of the easiness of items. This indicates that $\mathrm{ABaCo}$ has its highest discriminative power at a lower ability level and is not suitable for discriminating between normal individuals in the high range of performance.

Interrater reliability was evaluated on 45 randomly selected participants ( $15 \%$ of the total sample). The level of agreement among scores assigned by the two independent judges was calculated using the Intraclass Correlation Coefficient (ICC), which provided a generalized measure of interrater concordance adjusted for chance agreement between measurements. The ICC was .89 , indicating almost perfect interrater agreement based on Altman's (1991) indications.

Correlations among demographic variables and scores on the five $\mathrm{ABaCo}$ scales showed both age and education to be correlated with the majority of pragmatic scores. Gender 
Table 2 Statistical properties for age, education, and $\mathrm{ABaCo}$ subscales for each normative group

\begin{tabular}{|c|c|c|c|c|c|}
\hline \multirow[b]{2}{*}{ Age Groups } & \multicolumn{5}{|l|}{ Statistics } \\
\hline & Mean $(S D)$ & Median & Minimum-Maximum & Skewness & Kurtosis \\
\hline \multicolumn{6}{|l|}{ Age group $15-34(N=99)$} \\
\hline \multicolumn{6}{|l|}{ Education 8 years $(N=39)$} \\
\hline Age & $23.01(6.12)$ & 21.00 & $16-34$ & & \\
\hline Linguistic comprehension & $.91(.1)$ & .94 & $.65-1.00$ & -0.99 & 0.1 \\
\hline Linguistic production & $.86(.12)$ & .87 & $.64-1.00$ & -0.39 & -1.2 \\
\hline Extralinguistic comprehension & $.85(.12)$ & .86 & $.47-1.00$ & -0.95 & 1.47 \\
\hline Extralinguistic production & $.83(.13)$ & .85 & $.50-1.00$ & -0.63 & -0.27 \\
\hline Paralinguistic comprehension & $.88(.08)$ & .88 & $.67-1.00$ & -0.62 & 0.22 \\
\hline Paralinguistic production & $.96(.05)$ & .97 & $.84-1.00$ & -1.02 & -0.22 \\
\hline Context comprehension & $.85(.14)$ & .87 & $.44-1.00$ & -1.08 & 0.88 \\
\hline Context production & $.96(.11)$ & 1.00 & $.50-1.00$ & -2.96 & 8.91 \\
\hline Conversational scale & $.93(.1)$ & 1.00 & $.54-1.00$ & -1.83 & 3.87 \\
\hline \multicolumn{6}{|l|}{ Age group $15-34(N=99)$} \\
\hline \multicolumn{6}{|l|}{ Education 13 years $(N=45)$} \\
\hline Age & $28.02(3.67)$ & 29.00 & $20-34$ & & \\
\hline Linguistic comprehension & $.94(.08)$ & 1.00 & $.73-1.00$ & -1.32 & 0.9 \\
\hline Linguistic production & $.92(.08)$ & .94 & $.75-1.00$ & -0.67 & -0.44 \\
\hline Extralinguistic comprehension & $.94(.07)$ & .94 & $.75-1.00$ & -1.02 & 0.7 \\
\hline Extralinguistic production & $.83(.14)$ & .85 & $.43-1.00$ & -0.94 & 0.6 \\
\hline Paralinguistic comprehension & $.88(.06)$ & .90 & $.68-1.00$ & -0.54 & 1.08 \\
\hline Paralinguistic production & $.97(.04)$ & 1.00 & $.88-1.00$ & -1.49 & 1.14 \\
\hline Context comprehension & $.87(.13)$ & .90 & $.51-1.00$ & -1.01 & 0.45 \\
\hline Context production & $.96(.12)$ & 1.00 & $.50-1.00$ & -3.16 & 9.46 \\
\hline Conversational scale & $.96(.07)$ & 1.00 & $.75-1.00$ & -1.73 & 1.93 \\
\hline \multicolumn{6}{|l|}{ Age group $15-34(N=99)$} \\
\hline \multicolumn{6}{|l|}{ Education 17 years $(N=15)$} \\
\hline Age & $27.6(3.04)$ & 27.00 & $23-33$ & & \\
\hline Linguistic comprehension & $.97(.05)$ & 1.00 & $.83-1.00$ & -1.61 & 2.83 \\
\hline Linguistic production & $.95(.06)$ & 1.00 & $.83-1.00$ & -0.87 & -0.71 \\
\hline Extralinguistic comprehension & $.90(.08)$ & .92 & $.75-1.00$ & -0.27 & -1.13 \\
\hline Extralinguistic production & $.94(.08)$ & .98 & $.80-1.00$ & -0.74 & -1.15 \\
\hline Paralinguistic comprehension & $.92(.08)$ & .92 & $.71-1.00$ & -1.38 & 2.27 \\
\hline Paralinguistic production & $.98(.03)$ & 1.00 & $.94-1.00$ & -1.4 & 0.14 \\
\hline Context comprehension & $.94(.05)$ & .94 & $.88-1.00$ & .11 & -1.93 \\
\hline Context production & $.93(.11)$ & 1.00 & $.75-1.00$ & -1.18 & -0.73 \\
\hline Conversational scale & $.96(.06)$ & 1.00 & $.83-1.00$ & -1.05 & -0.36 \\
\hline \multicolumn{6}{|l|}{ Age group 35-54 $(N=106)$} \\
\hline \multicolumn{6}{|l|}{ Education 5 years $(N=6)$} \\
\hline Age & $45.33(8.26)$ & 46.50 & $35-53$ & & \\
\hline Linguistic comprehension & $.92(.09)$ & .94 & $.75-1.00$ & -1.27 & 1.53 \\
\hline Linguistic production & $.88(.12)$ & .92 & $.68-1.00$ & -0.87 & -0.53 \\
\hline Extralinguistic comprehension & $.81(.17)$ & .82 & $.51-1.00$ & -1.36 & 2.81 \\
\hline Extralinguistic production & $.88(.08)$ & .89 & $.75-.97$ & -0.80 & 0.33 \\
\hline Paralinguistic comprehension & $.79(.05)$ & .79 & $.71-.85$ & -0.26 & -1.67 \\
\hline Paralinguistic production & $.94(.07)$ & .97 & $.81-1.00$ & -1.54 & 2.13 \\
\hline Context comprehension & $.88(.08)$ & .92 & $.75-.94$ & -1.09 & -0.48 \\
\hline Context production & $.96(.10)$ & 1.00 & $.75-1.00$ & -1.09 & -0.48 \\
\hline
\end{tabular}


Table 2 (continued)

Statistics

\begin{tabular}{|c|c|c|c|c|c|}
\hline \multirow{2}{*}{ Age Groups } & \\
\hline & Mean $(S D)$ & Median & Minimum-Maximum & Skewness & Kurtosis \\
\hline \multicolumn{6}{|l|}{ Conversational scale } \\
\hline \multicolumn{6}{|l|}{ Age group $35-54(N=106)$} \\
\hline \multicolumn{6}{|l|}{ Education 8 years $(N=50)$} \\
\hline Age & 45.1 & 45.5 & $35-54$ & & \\
\hline Linguistic comprehension & $.90(.11)$ & .94 & $.57-1.00$ & -1.33 & 1.35 \\
\hline Linguistic production & $.87(.09)$ & .90 & $.67-1.00$ & -0.71 & -0.62 \\
\hline Extralinguistic comprehension & $.83(.13)$ & .83 & $.34-1.00$ & -1.02 & 2.41 \\
\hline Extralinguistic production & $.84(.11)$ & .86 & $.58-1.00$ & -0.43 & -0.54 \\
\hline Paralinguistic comprehension & $.84(.08)$ & .84 & $.57-1.00$ & -0.85 & 1.64 \\
\hline Paralinguistic production & $.95(.06)$ & .97 & $.75-1.00$ & -1.4 & 2.23 \\
\hline Context comprehension & $.87(.09)$ & .90 & $.63-1.00$ & -0.85 & 0.77 \\
\hline Context production & $.95(.10)$ & 1.00 & $.75-1.00$ & -1.55 & 0.41 \\
\hline Conversational scale & $.92(.09)$ & .96 & $.50-1.00$ & -1.90 & 6.59 \\
\hline \multicolumn{6}{|l|}{ Age group 35-54 $(N=106)$} \\
\hline \multicolumn{6}{|l|}{ Education 13 years $(N=35)$} \\
\hline Age & $43.17(6.09)$ & 42 & $35-54$ & & \\
\hline Linguistic comprehension & $.90(.09)$ & .94 & $.60-1.00$ & -1.33 & 1.89 \\
\hline Linguistic production & $.90(.09)$ & .92 & $.64-1.00$ & -1.05 & 0.80 \\
\hline Extralinguistic comprehension & $.81(.13)$ & .88 & $.59-1.00$ & -0.46 & -1.40 \\
\hline Extralinguistic production & $.86(.11)$ & .88 & $.61-1.00$ & -0.59 & -0.44 \\
\hline Paralinguistic comprehension & $.89(.09)$ & .92 & $.62-1.00$ & -1.28 & 1.71 \\
\hline Paralinguistic production & $.98(.04)$ & 1.00 & $.88-1.00$ & -1.54 & 1.16 \\
\hline Context comprehension & $.87(.12)$ & .88 & $.38-1.00$ & -2.23 & 7.44 \\
\hline Context production & $.94(.11)$ & 1.00 & $.75-1.00$ & -1.35 & -0.19 \\
\hline Conversational scale & $.97(.06)$ & 1.00 & $.75-1.00$ & -2.18 & 4.86 \\
\hline \multicolumn{6}{|l|}{ Age group $35-54(N=106)$} \\
\hline \multicolumn{6}{|l|}{ Education 17 years $(N=15)$} \\
\hline Age & $43.4(8.29)$ & 38 & $35-54$ & & \\
\hline Linguistic comprehension & $.93(.05)$ & .94 & $.83-1.00$ & -0.12 & -1.10 \\
\hline Linguistic production & $.90(.08)$ & .94 & $.73-1.00$ & -1.10 & 0.22 \\
\hline Extralinguistic comprehension & $.89(.09)$ & .92 & $.73-1.00$ & -0.55 & -0.69 \\
\hline Extralinguistic production & $.86(.13)$ & .88 & $.59-1.00$ & -0.76 & -0.10 \\
\hline Paralinguistic comprehension & $.90(.06)$ & .92 & $.76-.97$ & -0.80 & 0.11 \\
\hline Paralinguistic production & $.96(.04)$ & .97 & $.88-1.00$ & -1.15 & 0.38 \\
\hline Context comprehension & $.90(.06)$ & .88 & $.80-1.00$ & 0.18 & -0.48 \\
\hline Context production & $.92(.15)$ & 1.00 & $.50-1.00$ & -1.79 & 2.63 \\
\hline Conversational scale & $.94(.10)$ & 1.00 & $.63-1.00$ & -2.22 & 5.61 \\
\hline \multicolumn{6}{|l|}{ Age group 55-75 $(N=95)$} \\
\hline \multicolumn{6}{|l|}{ Education 5 years $(N=45)$} \\
\hline Age & $66.6(5.35)$ & 68 & $55-73$ & & \\
\hline Linguistic comprehension & $.87(.11)$ & .90 & $.61-1.00$ & -0.63 & -0.56 \\
\hline Linguistic production & $.85(.10)$ & .86 & $.59-1.00$ & -0.66 & -0.23 \\
\hline Extralinguistic comprehension & $.75(.15)$ & .77 & $.41-1.00$ & -0.37 & -0.01 \\
\hline Extralinguistic production & $.79(.12)$ & .81 & $.48-1.00$ & -0.31 & -0.32 \\
\hline Paralinguistic comprehension & $.80(.11)$ & .81 & $.51-.96$ & -0.65 & 0.08 \\
\hline Paralinguistic production & $.93(.09)$ & .94 & $.59-1.00$ & -1.84 & 4.56 \\
\hline Context comprehension & $.84(.12)$ & .88 & $.43-1.00$ & -1.17 & 1.62 \\
\hline
\end{tabular}


Table 2 (continued)

\begin{tabular}{|c|c|c|c|c|c|}
\hline \multirow[b]{2}{*}{ Age Groups } & \multicolumn{5}{|l|}{ Statistics } \\
\hline & Mean $(S D)$ & Median & Minimum-Maximum & Skewness & Kurtosis \\
\hline Context production & $.91(.18)$ & 1.00 & $.25-1.00$ & -2.12 & 4.14 \\
\hline Conversational scale & $.97(.07)$ & 1.00 & $.75-1.00$ & -2.07 & 3.17 \\
\hline \multicolumn{6}{|l|}{ Age group 55-75 $(N=95)$} \\
\hline \multicolumn{6}{|l|}{ Education 8 years $(N=20)$} \\
\hline Age & $63.1(5.35)$ & 63 & $55-71$ & & \\
\hline Linguistic comprehension & $.92(.08)$ & .94 & $.75-1.00$ & -0.78 & -0.48 \\
\hline Linguistic production & $.88(.10)$ & .90 & $.67-1.00$ & -0.89 & -0.04 \\
\hline Extralinguistic comprehension & $.85(.12)$ & .87 & $.59-1.00$ & -0.50 & -0.68 \\
\hline Extralinguistic production & $.85(.11)$ & .87 & $.61-.98$ & -0.61 & -0.23 \\
\hline Paralinguistic comprehension & $.81(.11)$ & .80 & $.63-1.00$ & 0.04 & -0.72 \\
\hline Paralinguistic production & $.94(.06)$ & .97 & $.81-1.00$ & -0.67 & -1.02 \\
\hline Context comprehension & $.88(.13)$ & .92 & $.39-1.00$ & -3.23 & 12.21 \\
\hline Context production & $.83(.24)$ & 1.00 & $.25-1.00$ & -1.05 & -0.21 \\
\hline Conversational scale & $.95(.09)$ & 1.00 & $.75-1.00$ & -1.66 & 1.42 \\
\hline \multicolumn{6}{|l|}{ Age group 55-75 $(N=95)$} \\
\hline \multicolumn{6}{|l|}{ Education 13 years $(N=15)$} \\
\hline Age & $62.4(5.97)$ & 60 & $55-72$ & & \\
\hline Linguistic comprehension & $.92(.07)$ & .94 & $.77-1.00$ & -0.81 & 0.51 \\
\hline Linguistic production & $.91(.08)$ & .92 & $.72-1.00$ & -0.79 & 0.31 \\
\hline Extralinguistic comprehension & $.83(.11)$ & .83 & $.66-1.00$ & -0.24 & -0.97 \\
\hline Extralinguistic production & $.85(.13)$ & .81 & $.67-1.00$ & -0.11 & -1.86 \\
\hline Paralinguistic comprehension & $.80(.13)$ & .84 & $.48-.96$ & -1.21 & 1.10 \\
\hline Paralinguistic production & $.97(.04)$ & 1.00 & $.88-1.00$ & -1.32 & 0.95 \\
\hline Context comprehension & $.91(.07)$ & .94 & $.75-1.00$ & -0.83 & 1.12 \\
\hline Context production & $.92(.15)$ & 1.00 & $.50-1.00$ & -1.79 & 2.63 \\
\hline Conversational scale & $.99(.01)$ & 1.00 & $.96-1.00$ & -2.41 & 4.35 \\
\hline \multicolumn{6}{|l|}{ Age group 55-75 $(N=95)$} \\
\hline \multicolumn{6}{|l|}{ Education 17 years $(N=15)$} \\
\hline Age & $58.39(4.87)$ & 56 & $55-72$ & & \\
\hline Linguistic comprehension & $.91(.06)$ & .92 & $.81-1.00$ & -0.02 & -1.03 \\
\hline Linguistic production & $.83(.10)$ & .81 & $.68-1.00$ & 0.20 & -1.28 \\
\hline Extralinguistic comprehension & $.79(.12)$ & .78 & $.61-1.00$ & 0.36 & -0.80 \\
\hline Extralinguistic production & $.75(.15)$ & .81 & $.55-.97$ & -0.02 & -1.61 \\
\hline Paralinguistic comprehension & $.84(.09)$ & .86 & $.68-.96$ & -0.48 & -0.94 \\
\hline Paralinguistic production & $.99(.02)$ & 1.00 & $.94-1.00$ & -1.09 & 0.40 \\
\hline Context comprehension & $.88(.09)$ & .90 & $.70-1.00$ & -0.41 & -0.37 \\
\hline Context production & $.87(.19)$ & 1.00 & $.50-1.00$ & -1.07 & -0.11 \\
\hline Conversational scale & $.96(.05)$ & 1.00 & $.88-1.00$ & -0.58 & -1.4 \\
\hline
\end{tabular}

was significantly correlated only with extralinguistic production and context scale comprehension (see Table 3).

The relative effects of age, education, and sex on the $\mathrm{ABaCo}$ scores were further investigated by performing regression analyses where each variable was entered separately, taking each subscale score as the dependent variable and age, education, (years of schooling), and sex as the independent ones. This procedure allowed us to evaluate the effect of each demographic variable within the regression model by partially removing the common effect. The results are shown in Table 4 .

Since participants' scores were affected mainly by age and years of education, the norms were stratified by these two variables. Table 5 shows the normative data for the $\mathrm{ABaCo}$ scales.

The data we collected can also be used to forecast individual performance scores. Having estimated a regression equation from the observed cases in the data set, the same 
Fig. 1 Distribution of scores across the nine $\mathrm{ABaCo}$ scales (considering the entire normative sample)
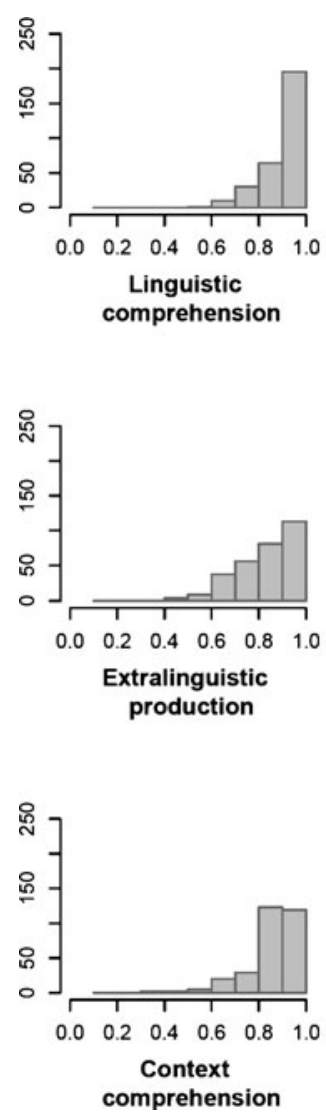
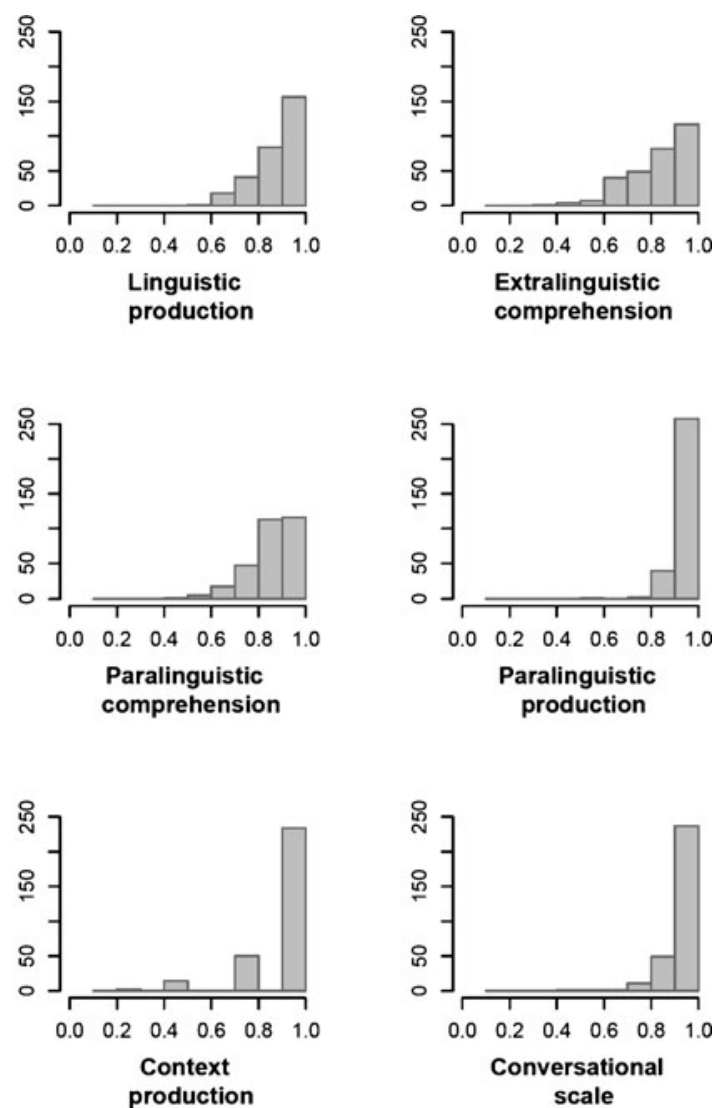

equation can be used to predict the value of the dependent variable $Y$ for new observations. Equation 1 provides a point estimate $\widehat{Y}_{i}$ of the expected value of $Y$ given the age and education of individual $i$ :

$\widehat{Y}_{i}=B_{0}+B_{\text {age }} X_{i, \text { age }}+B_{\text {education }} X_{i, \text { education }}$
In addition, confidence intervals can be computed around the point estimate, in order to evaluate whether a new observation (i.e., an individual's actual $Y$ score) is atypical with respect to the normative sample. The $s d$ of the distance between the point estimate $\widehat{Y}_{i}$ for individual $i$ and his/her actual score $Y_{i}$ is given by Eq. 2 :

$s d_{Y_{i}-\widehat{Y}_{i}}=\frac{S E_{Y-\widehat{Y}}}{\sqrt{n}} \sqrt{n+1+\frac{z_{i, \text { age }}^{2}}{1-R_{\text {age }}^{2}}+\frac{z_{i, \text { education }}^{2}}{1-R_{\text {education }}^{2}}-2 \frac{\beta_{\text {education, age }} z_{i, \text { age }} z_{\text {i,education }}}{1-R_{\text {education }}^{2}}}$,

where $z$ represents an individual's standardized age and education scores and $\beta_{\text {education, age }}$ is the standardized regression coefficient of education on age. The relevant values for each dependent variable are given in Appendix 3. The desired confidence intervals can easily be obtained by multiplying $s d_{Y_{i}-\widehat{Y}_{i}}$ by the appropriate coefficient (e.g., \pm 1.96 for 95\% CIs).

\section{Discussion}

The results from the present study provide norms for the nine scales of the $\mathrm{ABaCo}$, a clinical battery for the assessment of communicative abilities. These norms add to the validity of the battery as a valuable aid for the assessment of communicative outcome in patients with cerebral lesions or suffering from neuropsychiatric disorders. The main clinical utility of these norms is that they will give neuropsychologists/speech therapists the opportunity to more precisely determine the degree to which communicative abilities are impaired in patients of different ages and educational levels, by comparing their scores against those achieved by the corresponding normative group.

Providing norms for each of the nine $\mathrm{ABaCo}$ scales offers the additional advantage of allowing the clinician to determine whether or not one specific communicative modality is 
Table 3 Correlations of age, education, and gender with $\mathrm{ABaCo}$ scales

\begin{tabular}{llll}
\hline & Age & Education & Gender \\
\hline Linguistic comprehension & $-.154^{* *}$ & $.191^{* *}$ & .093 \\
Linguistic production & $-.162^{* *}$ & $.195^{* *}$ & .018 \\
Extralinguistic comprehension & $-.318^{* *}$ & $.229^{* *}$ & .011 \\
Extralinguistic production & $-.140^{*}$ & .099 & $.126^{*}$ \\
Paralinguistic comprehension & $-.378^{* *}$ & $.262^{* *}$ & -.025 \\
Paralinguistic production & $-.180^{* *}$ & $.286^{* *}$ & .009 \\
Context comprehension & -.019 & $.152^{*}$ & $-.130^{*}$ \\
Context production & $-.212^{* *}$ & .002 & -.091 \\
Conversational & .057 & .045 & -.074 \\
\hline
\end{tabular}

$* p<.05$

$* * p<.01$ affected with respect to others. For example, distinct clinical profiles have been described in a population of right-hemisphere-damaged patients; specifically, prosodic impairments, conversational discourse disorders, and emotional prosody have been found to dissociate (Benton \& Bryan, 1996). Individuals with TBI present with symptoms ranging from mild to severe, and the outcomes may vary considerably between patients. As a result, patients point out some strengths and some weaknesses, and marked interindividual differences have been noted among patients (Dardier et al., 2011; Lê, Coelho, Mozeiko, Krueger, \& Grafman, 2011), as well as specific domains of communicative impairment (Cannizzaro \& Coelho, 2002; Ehrlich \& Sipes, 1985). Alternatively, the clinician can select and administer only a certain set of scales, depending on the patient's specific needs. The results of this article thus extend the clinical utility and validity of the $\mathrm{ABaCo}$, making clinicians more

Table 4 Regression analyses predicting performance on each $\mathrm{ABaCo}$ scale from age, education, and sex

\begin{tabular}{|c|c|c|c|c|c|c|}
\hline DVs & IVs & $R_{\mathrm{Adj}}^{2}$ & $\Delta R^{2}$ & $F$ & $\beta$ & $95 \% \mathrm{CI}$ \\
\hline \multirow[t]{3}{*}{ Linguistic comprehension } & Age & .020 & .024 & $7.187 * *$ & $-.153 * *$ & {$[-.001, .000]$} \\
\hline & Education & .040 & .023 & $7.177 * *$ & $.158^{* *}$ & {$[.001, .006]$} \\
\hline & Sex & .044 & .008 & $5.6^{* *}$ & .087 & {$[-.004, .037]$} \\
\hline \multirow[t]{3}{*}{ Linguistic production } & Age & .026 & .026 & $7.814 * *$ & $-.16^{* *}$ & {$[-.002, .000]$} \\
\hline & Education & .049 & .023 & $7.635^{* *}$ & $.161^{* *}$ & {$[.001, .007]$} \\
\hline & Sex & .049 & .000 & $5.085^{* *}$ & .01 & {$[-.02, .024]$} \\
\hline \multirow[t]{3}{*}{ Extralinguistic comprehension } & Age & .099 & .102 & $33.69 * *$ & $-.319 * *$ & {$[-.003,-.002]$} \\
\hline & Education & .114 & .019 & $20.28 * *$ & $.144 *$ & {$[.001, .008]$} \\
\hline & Sex & .111 & .000 & $13.19^{* *}$ & .011 & {$[-.025, .031]$} \\
\hline \multirow[t]{3}{*}{ Extralinguistic production } & Age & .016 & .019 & $5.855^{*}$ & $-.139 *$ & {$[-.002, .000]$} \\
\hline & Education & .016 & .003 & $3.446^{*}$ & .061 & {$[-.002, .006]$} \\
\hline & Sex & .029 & .016 & $3.991 * *$ & $.127 *$ & {$[.004, .060]$} \\
\hline \multirow[t]{3}{*}{ Paralinguistic comprehension } & Age & .142 & .145 & $50.535^{* *}$ & $-.381 * *$ & {$[-.003,-.002]$} \\
\hline & Education & .162 & .023 & $29.9 * *$ & $.158 * *$ & {$[.001, .006]$} \\
\hline & Sex & .160 & .001 & $20.005^{* *}$ & -.031 & {$[-.026, .014]$} \\
\hline \multirow[t]{3}{*}{ Paralinguistic production } & Age & .030 & .033 & $10.225^{* *}$ & $-.182 * *$ & {$[-.001, .000]$} \\
\hline & Education & .085 & .058 & $14.917 * *$ & $.254 * *$ & {$[.002, .005]$} \\
\hline & Sex & .082 & .000 & $9.911 * * *$ & .001 & {$[-.012, .012]$} \\
\hline \multirow[t]{3}{*}{ Context comprehension } & Age & -.003 & .000 & .119 & -.02 & {$[.000, .001]$} \\
\hline & Education & .017 & .023 & $3.624 *$ & $.161 * *$ & {$[.001, .008]$} \\
\hline & Sex & .027 & .013 & $3.739^{*}$ & $-.113^{*}$ & {$[-.051, .000]$} \\
\hline \multirow[t]{3}{*}{ Context production } & Age & .041 & .045 & $13.884 * *$ & $-.211 * *$ & {$[-.003, .000]$} \\
\hline & Education & .043 & .005 & $7.658 * *$ & -.071 & {$[-.007, .002]$} \\
\hline & Sex & .048 & .009 & $6.048 * *$ & -.093 & {$[-.059, .005]$} \\
\hline \multirow[t]{3}{*}{ Conversational scale } & Age & .000 & .003 & 1.017 & .058 & {$[.000, .001]$} \\
\hline & Education & .001 & .004 & 1.168 & .07 & {$[-.001, .004]$} \\
\hline & Sex & .003 & .005 & 1.316 & .073 & {$[-.030, .006]$} \\
\hline
\end{tabular}

Note. $N=300 . \mathrm{DV}$, dependent variable; IV, independent variable; $\mathrm{CI}$, confidence interval

$* p<.05$

$* * p<.01$ 
Table 5 Percentiles for $\mathrm{ABaCo}$ scales scores for each normative group

\begin{tabular}{lllll}
\hline Percentile & Education & Education & Education & Education \\
& 5 Years & 8 Years & 13 Years & $17+$ Years
\end{tabular}

Linguistic Comprehension
Age group 15-34 $(N=99)$

90

80

70

60

50

40

30

20

10

Age group 35-54 $(N=106)$

$\begin{array}{ll}90 & 1.00 \\ 80 & 1.00 \\ 70 & .99 \\ 60 & .95 \\ 50 & .94 \\ 40 & .92 \\ 30 & .88 \\ 20 & .80 \\ 10 & .75\end{array}$

Age group 55-75 $(N=95)$

$\begin{array}{lllll}90 & 1.00 & 1.00 & 1.00 & 1.00 \\ 80 & .96 & 1.00 & .99 & .99 \\ 70 & .93 & 1.00 & .94 & .94 \\ 60 & .93 & .98 & .94 & .93 \\ 50 & .90 & .94 & .94 & .92 \\ 40 & .87 & .92 & .91 & .89 \\ 30 & .81 & .87 & .89 & .87 \\ 20 & .76 & .84 & .87 & .84 \\ 10 & .72 & .77 & .79 & .81\end{array}$

Linguistic Production

Age group 15-34 $(N=99)$

$\begin{array}{lllll}90 & - & 1.00 & 1.00 & 1.00 \\ 80 & - & .98 & 1.00 & 1.00 \\ 70 & - & .94 & .98 & 1.00 \\ 60 & - & .94 & .94 & 1.00 \\ 50 & - & .87 & .94 & 1.00 \\ 40 & - & .81 & .91 & .94 \\ 30 & - & .81 & .87 & .93 \\ 20 & - & .72 & .85 & .88 \\ 10 & - & .69 & .81 & .84\end{array}$

Age group 35-54 $(N=106)$

$\begin{array}{lllll}90 & 1.00 & .97 & .99 & .99 \\ 80 & .99 & .94 & .98 & .97 \\ 70 & .98 & .94 & .96 & .95 \\ 60 & .97 & .92 & .94 & .94 \\ 50 & .92 & .90 & .92 & .94\end{array}$

Table 5 (continued)

\begin{tabular}{lllll}
\hline Percentile & $\begin{array}{l}\text { Education } \\
5 \text { Years }\end{array}$ & $\begin{array}{l}\text { Education } \\
8 \text { Years }\end{array}$ & $\begin{array}{l}\text { Education } \\
\text { 13 Years }\end{array}$ & $\begin{array}{l}\text { Education } \\
17+\text { Years }\end{array}$ \\
\hline 40 & .86 & .87 & .89 & .92 \\
30 & .80 & .83 & .87 & .87 \\
20 & .73 & .76 & .81 & .81 \\
10 & .68 & .73 & .76 & .75 \\
Age group $55-75(N=95)$ & & & \\
90 & .97 & 1.00 & 1.00 & .98 \\
80 & .94 & .97 & 1.00 & .94 \\
70 & .92 & .96 & .97 & .90 \\
60 & .89 & .93 & .94 & .87 \\
50 & .86 & .90 & .92 & .81 \\
40 & .83 & .87 & .89 & .78 \\
30 & .81 & .86 & .87 & .75 \\
20 & .75 & .77 & .83 & .72 \\
10 & .69 & .68 & .77 & .69
\end{tabular}

Extralinguistic Comprehension

Age group 15-34 $(N=99)$

$\begin{array}{lllll}90 & - & 1.00 & 1.00 & 1.00 \\ 80 & - & .96 & 1.00 & 1.00 \\ 70 & - & .92 & 1.00 & .95 \\ 60 & - & .89 & .98 & .93 \\ 50 & - & .86 & .94 & .92 \\ 40 & - & .83 & .92 & .88 \\ 30 & - & .81 & .92 & .83 \\ 20 & - & .75 & .90 & .81 \\ 10 & - & .68 & .85 & .77 \\ \text { Age group } 35-54(N=106) & & & \\ 90 & 1.00 & 1.00 & .94 & 1.00 \\ 80 & .97 & .96 & .94 & .99 \\ 70 & .91 & .91 & .92 & .94 \\ 60 & .85 & .87 & .87 & .93 \\ 50 & .82 & .83 & .87 & .92 \\ 40 & .81 & .81 & .82 & .87 \\ 30 & .81 & .76 & .69 & .86 \\ 20 & .63 & .71 & .63 & .80 \\ 10 & .51 & .68 & .61 & .73\end{array}$

Age group 55-75 $(N=95)$

$\begin{array}{lllll}90 & .96 & 1.00 & .99 & .99\end{array}$

$\begin{array}{lllll}80 & .90 & .98 & .93 & .91\end{array}$

$\begin{array}{lllll}70 & .82 & .92 & .90 & .86\end{array}$

$\begin{array}{lllll}60 & .79 & .91 & .88 & .81\end{array}$

$\begin{array}{lllll}50 & .77 & .87 & .82 & .78\end{array}$

$\begin{array}{lllll}40 & .72 & .81 & .81 & .73\end{array}$

$\begin{array}{lllll}30 & .69 & .79 & .79 & .70\end{array}$

$\begin{array}{lllll}20 & .66 & .73 & .70 & .68\end{array}$

$\begin{array}{lllll}10 & .55 & .64 & .67 & .62\end{array}$

Extralinguistic Production

Age group 15-34 $(N=99)$

90

$1.00 \quad 1.00$

1.00 
Table 5 (continued)

\begin{tabular}{lllll}
\hline Percentile & $\begin{array}{l}\text { Education } \\
\text { 5 Years }\end{array}$ & $\begin{array}{l}\text { Education } \\
8 \text { Years }\end{array}$ & $\begin{array}{l}\text { Education } \\
13 \text { Years }\end{array}$ & $\begin{array}{l}\text { Education } \\
17+\text { Year }\end{array}$ \\
\hline 80 & - & .94 & .94 & 1.00 \\
70 & - & .94 & .94 & 1.00 \\
60 & - & .92 & .88 & 1.00 \\
50 & - & .85 & .85 & .98 \\
40 & - & .81 & .82 & .94 \\
30 & - & .75 & .77 & .86 \\
20 & - & .75 & .71 & .86 \\
10 & - & .63 & .63 & .81
\end{tabular}

Age group $35-54(N=106)$

$\begin{array}{lllll}90 & .97 & 1.00 & .99 & 1.00 \\ 80 & .96 & .94 & .97 & 1.00 \\ 70 & .95 & .92 & .94 & .95 \\ 60 & .92 & .87 & .92 & .93 \\ 50 & .89 & .86 & .87 & .87 \\ 40 & .87 & .81 & .83 & .83 \\ 30 & .84 & .76 & .81 & .79 \\ 20 & .79 & .73 & .76 & .77 \\ 10 & .75 & .69 & .69 & .63\end{array}$

Age group 55-75 $(N=95)$

$\begin{array}{lll}90 & .94 & .98 \\ 80 & .90 & .98 \\ 70 & .87 & .92 \\ 60 & .83 & .87 \\ 50 & .81 & .87 \\ 40 & .75 & .83 \\ 30 & .72 & .81 \\ 20 & .69 & .75 \\ 10 & .63 & .66\end{array}$

Paralinguistic Comprehension

Age group 15-34 $(N=99)$

$\begin{array}{ll}90 & - \\ 80 & - \\ 70 & - \\ 60 & - \\ 50 & - \\ 40 & - \\ 30 & - \\ 20 & - \\ 10 & -\end{array}$

Age group 35-54 $(N=106)$

$\begin{array}{ll}90 & .85 \\ 80 & .84 \\ 70 & .83 \\ 60 & .82 \\ 50 & .79 \\ 40 & .76 \\ 30 & .75 \\ 20 & .73\end{array}$

\subsection{0}

.98

.97

.96

.81

.78

.75

.72

.67

$$
.90
$$$$
.90
$$

.90

.86

.86

.82

.81

.95

.92

.90

.87

.84

.81

.81

.77

$\begin{array}{ll}1.00 & .97 \\ .96 & .96 \\ .96 & .93 \\ .94 & .92 \\ .92 & .92 \\ .88 & .89 \\ .87 & .86 \\ .84 & .85\end{array}$

.95

.91

.85

.81

.81

.67

.61

.58

.56

40

1.00

1.00

.97

.94

.92

.92

.91

.87

.77

.97

.96

.93

.92

.92

.89

.86
85
Table 5 (continued)

\begin{tabular}{lllll}
\hline Percentile & $\begin{array}{l}\text { Education } \\
5 \text { Years }\end{array}$ & $\begin{array}{l}\text { Education } \\
8 \text { Years }\end{array}$ & $\begin{array}{l}\text { Education } \\
13 \text { Years }\end{array}$ & $\begin{array}{l}\text { Education } \\
17+\text { Years }\end{array}$ \\
\hline 10 & .71 & .76 & .75 & .78
\end{tabular}

Age group 55-75 $(N=95)$

$\begin{array}{lllll}90 & .95 & 1.00 & .95 & .94 \\ 80 & .90 & .90 & .88 & .92 \\ 70 & .86 & .87 & .87 & .91 \\ 60 & .84 & .84 & .86 & .89 \\ 50 & .81 & .80 & .84 & .86 \\ 40 & .79 & .78 & .82 & .81 \\ 30 & .76 & .76 & .78 & .79 \\ 20 & .69 & .69 & .67 & .75 \\ 10 & .65 & .63 & .55 & .69\end{array}$

Paralinguistic Production

Age group 15-34 $(N=99)$

$\begin{array}{lllll}90 & - & 1.00 & 1.00 & 1.00 \\ 80 & - & 1.00 & 1.00 & 1.00 \\ 70 & - & 1.00 & 1.00 & 1.00 \\ 60 & - & 1.00 & 1.00 & 1.00 \\ 50 & - & .97 & 1.00 & 1.00 \\ 40 & - & .97 & 1.00 & 1.00 \\ 30 & - & .94 & .97 & .99 \\ 20 & - & .91 & .94 & .94 \\ 10 & - & .87 & .89 & .94\end{array}$

Age group 35-54 $(N=106)$

$\begin{array}{lllll}90 & 1.00 & 1.00 & 1.00 & 1.00 \\ 80 & .99 & 1.00 & 1.00 & 1.00 \\ 70 & .97 & 1.00 & 1.00 & 1.00 \\ 60 & .97 & .97 & 1.00 & 1.00 \\ 50 & .97 & .97 & 1.00 & .97 \\ 40 & .96 & .94 & 1.00 & .97 \\ 30 & .91 & .94 & .97 & .94 \\ 20 & .85 & .91 & .94 & .94 \\ 10 & .81 & .88 & .89 & .87\end{array}$

Age group 55-75 $(N=95)$

$\begin{array}{lllll}90 & 1.00 & 1.00 & 1.00 & 1.00 \\ 80 & 1.00 & 1.00 & 1.00 & 1.00 \\ 70 & 1.00 & 1.00 & 1.00 & 1.00 \\ 60 & .97 & 1.00 & 1.00 & 1.00 \\ 50 & .94 & .97 & 1.00 & 1.00 \\ 40 & .94 & .94 & .97 & .98 \\ 30 & .89 & .88 & .96 & .97 \\ 20 & .87 & .87 & .94 & .97 \\ 10 & .83 & .85 & .89 & .96\end{array}$

Context Comprehension

Age group 15-34 $(N=99)$

$\begin{array}{lllll}90 & - & 1.00 & 1.00 & 1.00 \\ 80 & - & 1.00 & 1.00 & 1.00 \\ 70 & - & .94 & .94 & 1.00 \\ 60 & - & .90 & .94 & .97\end{array}$


Table 5 (continued)

\begin{tabular}{lllll}
\hline Percentile & $\begin{array}{l}\text { Education } \\
\text { 5 Years }\end{array}$ & $\begin{array}{l}\text { Education } \\
8 \text { Years }\end{array}$ & $\begin{array}{l}\text { Education } \\
\text { 13 Years }\end{array}$ & $\begin{array}{l}\text { Education } \\
17+\text { Years }\end{array}$ \\
\hline 50 & - & .87 & .90 & .94 \\
40 & - & .87 & .84 & .90 \\
30 & - & .81 & .83 & .90 \\
20 & - & .75 & .78 & .88 \\
10 & - & .64 & .67 & .87
\end{tabular}

Age group 35-54 $(N=106)$

$\begin{array}{lll}90 & .94 & .99 \\ 80 & .94 & .94 \\ 70 & .94 & .92 \\ 60 & .94 & .90 \\ 50 & .92 & .90 \\ 40 & .88 & .87 \\ 30 & .82 & .81 \\ 20 & .77 & .80 \\ 10 & .75 & .75 \\ \text { Age group } 55-75(N=95) & \\ 90 & .96 & .99 \\ 80 & .94 & .94 \\ 70 & .94 & .94 \\ 60 & .89 & .94 \\ 50 & .87 & .92 \\ 40 & .81 & .88 \\ 30 & .81 & .87 \\ 20 & .75 & .87 \\ 10 & .69 & .76\end{array}$

Context Production

Age group 15-34 $(N=99)$

90
80
70
60
50
40
30
20
10

$\begin{array}{ll}- & 1.00 \\ - & 1.00 \\ - & 1.00 \\ - & 1.00 \\ - & 1.00 \\ - & 1.00 \\ - & 1.00 \\ - & 1.00 \\ - & .75\end{array}$

Age group 35-54 $(N=106)$

$\begin{array}{lcccc}90 & 1.00 & 1.00 & 1.00 & 1.00 \\ 80 & 1.00 & 1.00 & 1.00 & 1.00 \\ 70 & 1.00 & 1.00 & 1.00 & 1.00 \\ 60 & 1.00 & 1.00 & 1.00 & 1.00 \\ 50 & 1.00 & 1.00 & 1.00 & 1.00 \\ 40 & 1.00 & 1.00 & 1.00 & 1.00 \\ 30 & 1.00 & 1.00 & 1.00 & .95 \\ 20 & .85 & .80 & .75 & .75 \\ 10 & .75 & .75 & .75 & .65\end{array}$

Age group 55-75 $(N=95)$

90

1.00

1.00
Table 5 (continued)

\begin{tabular}{lllll}
\hline Percentile & $\begin{array}{l}\text { Education } \\
\text { 5 Years }\end{array}$ & $\begin{array}{l}\text { Education } \\
\text { 8 Years }\end{array}$ & $\begin{array}{l}\text { Education } \\
\text { 13 Years }\end{array}$ & $\begin{array}{l}\text { Education } \\
17+\text { Years }\end{array}$ \\
\hline 80 & 1.00 & 1.00 & 1.00 & 1.00 \\
70 & 1.00 & 1.00 & 1.00 & 1.00 \\
60 & 1.00 & 1.00 & 1.00 & 1.00 \\
50 & 1.00 & 1.00 & 1.00 & 1.00 \\
40 & 1.00 & .85 & 1.00 & .85 \\
30 & 1.00 & .75 & .95 & .75 \\
20 & .75 & .50 & .75 & .75 \\
10 & .65 & .50 & .65 & .50
\end{tabular}

Conversational Scale

Age group 15-34 $(N=99)$

$\begin{array}{lllll}90 & - & 1.00 & 1.00 & 1.00 \\ 80 & - & 1.00 & 1.00 & 1.00 \\ 70 & - & 1.00 & 1.00 & 1.00 \\ 60 & - & 1.00 & 1.00 & 1.00 \\ 50 & - & 1.00 & 1.00 & 1.00 \\ 40 & - & .94 & 1.00 & .97 \\ 30 & - & .92 & .96 & .92 \\ 20 & - & .83 & .92 & .88 \\ 10 & - & .75 & .83 & .86\end{array}$

Age group 35-54 $(N=106)$

$\begin{array}{llll}90 & 1.00 & 1.00 & 1.00\end{array}$

$\begin{array}{lllll}80 & 1.00 & 1.00 & 1.00 & 1.00\end{array}$

$\begin{array}{lllll}70 & 1.00 & 1.00 & 1.00 & 1.00\end{array}$

$\begin{array}{lllll}60 & 1.00 & 1.00 & 1.00 & 1.00\end{array}$

$\begin{array}{lllll}50 & 1.00 & .96 & 1.00 & 1.00\end{array}$

$\begin{array}{lllll}40 & 1.00 & .89 & 1.00 & .96\end{array}$

$\begin{array}{lllll}30 & 1.00 & .87 & .99 & .91\end{array}$

$\begin{array}{lllll}20 & 1.00 & .83 & .92 & .87\end{array}$

$\begin{array}{lllll}10 & 1.00 & .83 & .87 & .75\end{array}$

Age group 55-75 $(N=95)$

\begin{tabular}{lllll}
90 & 1.00 & 1.00 & 1.00 & 1.00 \\
80 & 1.00 & 1.00 & 1.00 & 1.00 \\
70 & 1.00 & 1.00 & 1.00 & 1.00 \\
60 & 1.00 & 1.00 & 1.00 & 1.00 \\
50 & 1.00 & 1.00 & 1.00 & 1.00 \\
40 & 1.00 & 1.00 & 1.00 & .93 \\
30 & 1.00 & 1.00 & 1.00 & .92 \\
20 & .92 & .87 & 1.00 & .92 \\
10 & .83 & .76 & .96 & .87 \\
\hline
\end{tabular}

confident in assessing impaired communicative abilities and planning treatment procedures.

In the present study, we provided norms for a sample of 300 healthy and cognitively intact individuals of different ages and educational levels. The sample used to develop the norms was recruited according to the ISTAT indications so as to be representative of the population from which it was 
drawn. Both age and education affected participants' scores; this suggests that these two variables have an impact on pragmatic performance even in the absence of neuropsychological disorders. In more detail, in line with another study by Zanini et al. (2005), we found that performance improved as age decreased and educational level increased. Consequently, the set of norms were stratified across age and educational level. In order to ensure the greatest clinical utility of the data presented in the presenst study, the values for the normative data are provided in Tables 2 and 5.

As is apparent from Table 2 and Fig. 1, the distribution of scores in the present normative sample was not normal. This is not surprising, given that $\mathrm{ABaCo}$ was designed for assessing pragmatic abilities in patients affected by communicative disorders and normal individuals should be expected to succeed in most of the tasks. When a battery is designed in such a way that the majority of normal individuals can succeed in most of the items, scores are compressed into values at the upper extreme of the score range, with only a few observations at the lower extreme. The resulting distribution is left-skewed, and the variability of scores falling within or above the normal range is limited. Consequently, the battery has its highest discriminative power at the lower ability levels, and it is most useful in identifying impaired individuals (for a discussion of the interpretation of scores not normally distributed in neuropsychological tests, see Mitrushina et al., 2005). The normative data obtained in the present study will be especially useful for a detailed assessment of patients affected by communication disorders, while they do not discriminate between normal individuals at high performance levels.

Moreover, to permit a more precise definition of each communicative outcome, we have also provided all the values needed to calculate the expected score for each patient on the basis of his/her age and educational level. This calculation is a valuable clinical tool, which will allow specialists to determine the distance between the patient's actual score and the expected score, automatically placing and quantifying the value on the basis of the percentile range. It will be sufficient to replace the normative values in formulas 1 and 2 for each scale provided in Appendix 3 and add the current age, educational level, and actual score of a particular patient to obtain that patient's expected score and the percentile below which he/she fell.

In sum, this study provides normative data for a new clinical battery for the assessment of communicative abilities $(\mathrm{ABaCo})$; in more detail, we have provided norms for each battery scale. Such norms will be useful for obtaining clinically indicative and reliable information, by making it possible to differentiate between real deficits and those attributable to the normal aging process or educational background, and will thus be helpful for designing specific treatment plans. Further research involving various clinical samples will continue to attest to the potential clinical utility and psychometric validation of this effective instrument.

Acknowledgments This research was supported by Regione Piemonte (IIINBEMA Project: Institutions, Behaviour and Markets in Local and Global Settings, and GIRS Project: The Invisible Grammar of Social Relations, Contract grant number L.R. n. 4/2006).

The authors would like to thank Filippo Migliorati, Chiara Abbas, Alessandra Agosti, Federica De Santis, Eliana Papa, and Laura Robiglio for their participation in data collection and Marco Del Giudice for his contribution to the statistical analysis.

\section{Appendix 1 ABaCo: Battery structure}

Table 6 Number and type of items for each scale

\begin{tabular}{lll}
\hline & Number of Items & Type of Item \\
\hline $\begin{array}{l}\text { I. Linguistic Scale } \\
\text { (1) Linguistic comprehension }\end{array}$ & \\
Basic & & \\
Assertion & 4 & \\
Question & 4 & Examiner's prompt \\
Request & 4 & Examiner's prompt \\
Command & 4 & Examiner's prompt \\
Standard & 4 & Examiner's prompt \\
Nonstandard & & Videotaped scene \\
Deceit & 4 & \\
Irony & 4 & Videotaped scene \\
(2) Linguistic production & & Videotaped scene \\
Basic & & \\
Assertion & 4 & \\
Question & 4 & Examiner's prompt \\
Request & 4 & Examiner's prompt \\
Command & 4 & Examiner's prompt \\
Standard & 4 & Examiner's prompt \\
Nonstandard & & Videotaped scene \\
Deceit & 4 & Videotaped scene \\
Irony & 4 & Videotaped scene \\
II. Extratisti Scale &
\end{tabular}

II. Extralinguistic Scale

(3) Extralinguistic comprehension Basic

$\begin{array}{lll}\text { Assertion } & 4 & \text { Examiner's prompt } \\ \text { Question } & 4 & \text { Examiner's prompt } \\ \text { Request } & 4 & \text { Examiner's prompt } \\ \text { Command } & 4 & \text { Examiner's prompt } \\ \text { Standard } & 4 & \text { Videotaped scene } \\ \text { Nonstandard } & & \\ \text { Deceit } & 4 & \text { Videotaped scene } \\ \text { Irony } & 4 & \text { Videotaped scene }\end{array}$


Table 6 (continued)

Number of Items $\quad$ Type of Item

(4) Extralinguistic production

Basic

$\begin{array}{lcl}\text { Assertion } & 4 & \text { Examiner's prompt } \\ \text { Question } & 4 & \text { Examiner's prompt } \\ \text { Request } & 4 & \text { Examiner's prompt } \\ \text { Command } & 4 & \text { Examiner's prompt } \\ \text { Standard } & 4 & \text { Videotaped scene } \\ \text { Nonstandard } & \\ \text { Deceit } & 4 & \text { Videotaped scene } \\ \text { Irony } & 4 & \text { Videotaped scene } \\ \text { III. Paralinguistic Scale } & & \\ \text { (5) Paralinguistic comprehension } & \\ \text { Basic } & 8 & \text { Videotaped scene } \\ \text { Emotion } & 8 & \text { Videotaped scene } \\ \text { Contradiction } & 4 & \text { Videotaped scene } \\ \text { (6) Paralinguistic production } & \\ \text { Basic } & 8 & \text { Examiner's prompt } \\ \text { Emotion } & 8 & \text { Examiner's prompt } \\ \text { IV. Context Scale } & & \\ \text { (7) Context scale comprehension } & 8 & \\ \text { Social norms } & 8 & \text { Examiner's prompt } \\ \text { Discourse norms } & 2 & \\ \text { (8) Context scale production } & & \\ \text { Formal } & & \\ \text { Informal } & & \text { Videotaped scene } \\ \text { V. Conversational Scale } & & \\ \text { (9) Comprehension/Production } & \\ \text { Topic/Turn taking } & 4 & \\ & & \\ & & \end{array}$

\section{Appendix 2 ABaCo: Item examples}

\section{(1) Linguistic Scale}

\section{Comprehension tasks}

Bsa: Assertion

Item in vivo \#2:

Examiner: "That wall is painted in white."

Test question: Tell me if the sentence I told you is true or false.

\section{Standard Communication Acts}

Videotaped item \#1:

Frank, Paula and Clare are in the kitchen, seated at the table, and they are having dinner. Frank says: "This pasta is very good, who has cooked it?" Paula answers: "I'm glad that my effort is appreciated"

Test question: Who cooked the pasta?

\section{Nonstandard Communication Acts: Irony}

Videotaped item \#9:

In a shop, Lara tries on a dress that is clearly too tight and asks Simon: "Does this dress fit me?" Simon answers: "I see your diet works!"

Test question: In your opinion, what did the boy want to say to the girl?

If the participant repeats the actor's reply: What does it mean?

In-depth question: Was he serious?

In-depth question: Why did the boy answer in this way?

\section{Production tasks}

\section{Standard Communication Acts}

Videotaped item \#13:

Husband and wife are sitting on the sofa. Wife: "What would you like to do this afternoon?"

Test question: What could the husband reply to the wife?

If the answer is not clear: What does it mean?

\section{Nonstandard Communication Acts: Deceit \\ Videotaped item \#17:}

Richard is in the bathroom. He inadvertently pours the perfume of his sister. He hastily dries and goes away. Sometime later his sister, with the empty bottle in her hands: "Who has poured my perfume?"

Test question: Richard doesn't want to be discovered. What could he answer?

If the answer is not clear: What does it mean?

\section{(2) Extralinguistic Scale}

\section{Comprehension tasks}

Bsa: Request

Videotaped item \#41:

The actress is standing on a chair next to a library and she is rearranging the books. A book falls from her hands and, looking at the camera, she points it as if asking the viewer to pick it up.

Test question: In your opinion, what did she want to say?

\section{Nonstandard Communication Acts: Deceit \\ Videotaped item \#53:}

Henry is sitting at the desk with books and exercise books, but instead of studying he is watching TV. Suddenly he hears his mother coming and he switches off TV with the remote control. His mother comes in the room and looks at him with an interrogative expression as if asking "What are you doing?" Henry spreads his hands innocently, as if saying: "Nothing".

Test question: In your opinion, what did the boy want to say to his mother?

If the participant repeats the actor's reply: What does it mean? 
In-depth question: Was he serious?

In-depth question: Why did the boy answer in that way to his mother?

\section{Production tasks}

\section{Standard Communication Acts}

Videotaped item \#59:

On a desolate country road, Deacon's car has broken down. Deacon seems to be there for a long time (he continuously watches at the clock, puts his hand over his eyes as if looking if someone is coming, he is nervous). Finally, he sees a car arriving.

Test question: The boy needs help. What gesture can be used?

If the answer is not clear: What does it mean?

\section{(3) Paralinguistic Scale}

\section{Comprehension tasks}

\section{Basic Emotion}

Videotaped item \#77:

The actor is scared.

Test question: In your opinion, what emotion is he communicating? How does he feel?

- sad

- $\quad$ scared (target)

- happy

- calm

\section{Paralinguistic contradiction}

Videotaped item \#80.

It's Robert's birthday. Monica gives him a gift. Monica: "Happy Birthday!" Robert opens the package and finds a tie with terrible colors. Showing bored face and voice, he says: "Thanks. Really, I really needed it . . beautiful! "

Test question: In your opinion, what did the boy want to say to the girl?

If the participant repeats the actor's reply: What does it mean?

In-depth question: In your opinion, did the boy like the tie? Why?

\section{Production tasks}

\section{Basic Speech Acts: Question, Command}

Item in vivo \#1.

Examiner: "Give me the pen."

Test question \#1: Try to ask me.

Test question \#2: Try to order me.

\section{Basic Emotion: Sadness, Joy}

Item in vivo \#7:

Examiner: "Ask me where the doctor is."
Test question \#1: Acting sad.

Test question \#2: Acting happy.

\section{(4) Context Scale}

\section{Comprehension tasks}

Discourse norms-Grice's maxims (quantity)

Videotaped item \#87:

Sister: "Where did you put my diary?" Brother, in front of a red chest of drawers: "In the red drawer."

Test question: Do you think the answer is correct?

If the patient says no: Why?

\section{Social norms}

Videotaped item \#84:

The head office: "Miss, could you please type this letter?" The secretary replies with an angry tone: "Now I cannot! I have a lot of work!"

Test question: Do you think the secretary has been polite? If the patient says no: Why?

\section{Production tasks}

Discourse norms

Item in vivo \#2:

Examiner: "Imagine you are late for an appointment

- with your lawyer

- with a friend of yours"

Test question: How would you apologize for being late?

\section{(5) Conversational Scale}

\section{Topic \#1 - Free-time}

Item in vivo \#4:

Free conversation between participant and examiner. Some suggestions for the examiner:

- What do you like to do in your free time?

- I really enjoy sport/reading/looking after my garden ...

Depending on how the conversation proceeds:

- What sort of books do you like reading?

- Which sports do you like practicing?

- Which sports do you like watching on television?

- I am passionate about cars/football/stamp-collecting . . .

- Which team do you support?

\section{Appendix 3 Relevant values to predict individual scores on all the battery scales}

Table 7 displays the relevant values to calculate individual's standardized age and education scores ( $z$ score). 
Table 7 Mean and standard deviations of age and education in the normative sample

\begin{tabular}{lllll}
\hline & $M_{\text {age }}$ & $S D_{\text {age }}$ & $M_{\text {education }}$ & $S D_{\text {education }}$ \\
\hline$n=300$ & 44.44 & 16.41 & 10.42 & 3.97 \\
\hline
\end{tabular}

For example, if an individual $i$ is 50 years of age and his/ her education is 13 years of schooling, the $z$ scores will be calculated as follows:

$z_{\text {age }}=\frac{i_{\text {age }}-M_{\text {age }}}{S D_{\text {age }}}=\frac{50-44.4}{16.41}=0.341$

$z_{\text {education }}=\frac{i_{\text {education }}-M_{\text {education }}}{S D_{\text {education }}}=\frac{13-10.42}{3.97}=0.65$

The standardized regression coefficient of education on age $\left(\beta_{\text {education, age }}\right)$ from the normative sample $(n=300)$ is

$\beta_{\text {education, age }}=-.31$

Table 8 displays the relevant values that can be used to forecast individual performance scores on all the battery scales.

Table 8 Relevant values derived from the regression analysis performed on each battery scale (scale scores as dependent variables; age and education as predictors)

\begin{tabular}{llllll}
\hline & $R^{2}$ & $S E$ & $\beta_{\text {age }}$ & $\beta_{\text {education }}$ & Intercept \\
\hline Linguistic comprehension & .046 & .091 & -.104 & .158 & 0.899 \\
Linguistic production & .049 & .095 & -.109 & .161 & 0.870 \\
Extralinguistic & .12 & .123 & -.274 & .144 & 0.888 \\
$\quad$ comprehension & & & & & \\
Extralinguistic production & .023 & .124 & -.120 & .061 & 0.857 \\
Paralinguistic comprehension & .168 & .088 & -.331 & .158 & 0.899 \\
Paralinguistic production & .091 & .054 & -.103 & .254 & 0.937 \\
Context scale comprehension & .024 & .112 & .031 & .161 & 0.814 \\
Context scale production & .049 & .142 & -.233 & -.071 & 1.048 \\
Conversational scale & .008 & .081 & .080 & .070 & 0.920 \\
\hline
\end{tabular}

Example: Fictitious individual $i$ aged 50 and his/her education is 13 years of schooling - Predicted value for linguistic comprehension scale.

Given Eqs. 6 and 7:

$\widehat{Y}_{i}=B_{0}+B_{\text {age }} X_{i, \text { age }}+B_{\text {education }} X_{i, \text { education }}$

we can predict the value of the dependent variable linguistic comprehension substituting the values given in the Eqs. 3 and 4 and in Table 8 for the linguistic comprehension scale into the Eq. 6:

$\widehat{Y}_{i}=0.899-0.104 * 0.341+0.158 * 0.65=0.966$.
We can also obtain the $s d$ of the distance between the point estimate $\widehat{Y}_{i}$ for individual $i$ and his/her actual score $Y_{i}$ substituting values in the Eq. 7 :

$s d_{Y_{i}-\widehat{Y}_{i}}=\frac{0.091}{\sqrt{300}} \sqrt{300+1+\frac{0.341^{2}}{1-0.046}+\frac{0.65^{2}}{1-0.046}-2 \frac{0.31 * 0.341 * 0.65}{1-0.046}}=0.091$.

The desired confidence intervals can be easily obtained by multiplying $s d_{Y_{i}-\widehat{Y}_{i}}$ by the appropriate coefficient (e.g., \pm 1.96 for $95 \%$ CIs).

\section{References}

Adams, C. (2002). Practitioner review: The assessment of language pragmatics. Journal of Child Psychology and Psychiatry, 43, 973-987.
Altman, D. G. (1991). Practical statistics for medical research. London: Chapman and Hall.

Angeleri, R., Bosco, F. M., Zettin, M., Sacco, K., Colle, L., \& Bara, B. G. (2008). Communicative impairment in traumatic brain injury: A complete pragmatic assessment. Brain and Language, 107, 229-245.

Bara, B. G. (2010). Cognitive pragmatics: The mental processes of communication. Cambridge: MIT Press.

Bara, B. G., Tirassa, M., \& Zettin, M. (1997). Neiropragmatics: Neuropsychological constraints on formal theories of dialogue. Brain and Language, 59, 7-49.

Bates, E. (1976). Language and context: Studies in the acquisition of pragmatics. New York: Academic Press. 
Benton, E., \& Bryan, K. (1996). Right cerebral hemisphere damage: Incidence of language problems. International Journal of Rehabilitation Research, 19, 47-54.

Bishop, D. V. M. (1998). Development of the children's communication checklist (CCC): A method for assessing qualitative aspects of communicative impairment in children. Journal of Child Psychology and Psychiatry, 39, 879-891.

Bryan, K. (1995). The Right Hemisphere Language Battery. London: Whurr.

Cannizzaro, M. S., \& Coelho, C. A. (2002). Treatment of story grammar following traumatic brain injury: A pilot study. Brain Injury, 16, 1065-1073.

Cutica, I., Bucciarelli, M., \& Bara, B. G. (2006). Neuropragmatics: Extralinguistic pragmatic ability is better preserved in lefthemisphere-damaged patients than in right-hemisphere-damaged patients. Brain and Language, 98, 12-25.

Dardier, V., Bernicot, J., Delanoë, A., Vanberten, M., Fayada, C., Chevignard, M., \& Dubois, B. (2011). Severe traumatic brain injury, frontal lesions, and social aspects of language use: A study of French-speaking adults. Journal of Communication Disorders, 44, 359-378.

Dennis, M., \& Barnes, M. A. (1990). Knowing the meaning, getting the point, bridging the gap, and carrying the message: Aspects of discourse following closed head injury in childhood and adolescence. Brain and Language, 39, 428-446.

Ehrlich, J. S., \& Sipes, A. L. (1985). Group treatment of communication skills for head trauma patients. Cognitive Rehabilitation, 3, 32-37.

Ferré, P., Ska, B., Lajoie, C., Bleau, A., \& Joanette, Y. (2011). Clinical focus on prosodic, discursive and pragmatic treatment for right hemisphere damaged adults: What's right? Rehabilitation Research and Practice. Advance online publication. doi:10.1155/2011/131820

Friedland, D., \& Miller, N. (1998). Conversation analysis of communication breakdown after closed head injury. Brain Injury, 12, 1-14.

Gardner, H., \& Brownell, H. H. (1986). Right Hemisphere Communication Battery. Boston: Psychology Service, VAMC.

Hartley, L. L., \& Jenson, P. J. (1992). Three discourse profiles of closed-head-injury speakers: Theoretical and clinical implications. Brain Injury, 6, 271-281.
Kempson, R. M. (1975). Presupposition and the delimitation of semantics. Cambridge: Cambridge University Press.

Lê, K., Coelho, C. A., Mozeiko, J., Krueger, F., \& Grafman, J. (2011). Measuring goodness of story narratives: Implications for traumatic brain injury. Aphasiology, 25, 748-760.

McDonald, S. (1993). Pragmatic language skills after closed head injury: Ability to meet the informational needs of the listener. Brain and Language, 44, 28-46.

McDonald, S., Flanagan, S., Rollins, J., \& Kinch, J. (2003). TASIT: A new clinical tool for assessing social perception after traumatic brain injury. The Journal of Head Trauma Rehabilitation, 18, 219-238.

Mitrushina, M., Boone, K. B., Razani, J., \& D’Elia, L. F. (2005). Handbook of normative data for neuropsychological assessment (2nd ed.). Oxford: Oxford University Press.

Penn, C. (1985). The profile of communicative appropriateness: A clinical tool for the assessment of pragmatics. South African Journal of Communication Disorders, 32, 18-23.

Prutting, C. A. (1982). Pragmatics as social competence. The Journal of Speech and Hearing Disorders, 47, 123-134.

Prutting, C. A., \& Kirchner, D. M. (1987). A clinical appraisal of the pragmatic aspects of language. The Journal of Speech and Hearing Disorders, 52, 105-119.

Sacco, K., Angeleri, R., Bosco, F. M., Colle, L., Mate, D., \& Bara, B. G. (2008). Assessment Battery for Communication-ABaCo: A new instrument for the evaluation of pragmatic abilities. Journal of Cognitive Science, 9, 111-157.

Snow, P., Douglas, J., \& Ponsford, J. (1998). Conversational discourse abilities following severe traumatic brain injury: A follow-up study. Brain Injury, 12, 911-935.

Turkstra, L. S., McDonald, S., \& Kaufmann, P. M. (1995). Assessment of pragmatic communication skills in adolescents after traumatic brain injury. Brain Injury, 10, 329-345.

Zanini, S., \& Bryan, K. (2003). La batteria del linguaggio dell'emisfero destro-BLED. Bologna: EMS.

Zanini, S., Bryan, K., De Luca, G., \& Bava, A. (2005). Italian Right Hemisphere Language Battery: The normative study. Neurological Science, 26, 13-25. 